\title{
Glycemic Index and Response of a Plant Based Nutritional Supplement and Its Subjective Satiety Following Its Use in Indian Adults
}

\author{
Bhoite Rachana ${ }^{*}$, Parthasarthy Vijayalakshmi², Raman Jeevan Ganesh ${ }^{2}$, Viswanathan Gopinath ${ }^{2}$, \\ Karthikeyan Parkavi², Vasudevan Kavitha², Nagamuthu Gayathri' ${ }^{2}$, Rajagopal Gayathri², \\ Shanmugam Shobana ${ }^{2}$, Ranjit Mohan Anjana ${ }^{3}$, Ranjit Unnikrishnan ${ }^{3}$, Vasudevan Sudha', \\ Mehta Suyog ${ }^{1}$, Archana Toppo Panda ${ }^{1}$, Acharya Shivani ${ }^{1}$, Viswanathan Mohan ${ }^{3}$ \\ ${ }^{1}$ Dr. Reddy's Laboratories, Hyderabad, India \\ ${ }^{2}$ Department of Foods, Nutrition \& Dietetics Research, Madras Diabetes Research Foundation, Chennai, India \\ ${ }^{3}$ Department of Diabetology, Dr. Mohan’s Diabetes Specialities Centre, Chennai, India \\ Email: *rachanamb@drreddys.com
}

How to cite this paper: Rachana, B., Vijayalakshmi, P., Ganesh, R.J., Gopinath, V., Parkavi, K., Kavitha, V., Gayathri, N., Gayathri, R., Shobana, S., Anjana, R.M., Unnikrishnan, R., Sudha, V., Suyog, M., Panda, A.T., Shivani, A. and Mohan, V. (2019) Glycemic Index and Response of a Plant Based Nutritional Supplement and Its Subjective Satiety Following Its Use in Indian Adults. Food and Nutrition Sciences, 10, 937-946.

https://doi.org/10.4236/fns.2019.108067

Received: June 18, 2019

Accepted: August 11, 2019

Published: August 14, 2019

Copyright $\odot 2019$ by author(s) and Scientific Research Publishing Inc. This work is licensed under the Creative Commons Attribution International License (CC BY 4.0).

http://creativecommons.org/licenses/by/4.0/

cc) (7) Open Access

\begin{abstract}
Background: Diet plays a vital role in managing diabetes. Foods with a low glycemic index provide lower postprandial glucose spikes and induce satiety. The objective of this study was to assess the Glycemic index (GI) without milk and Glycemic response (GR) with milk of two different flavours of a plant-based supplement which is high in protein and fibre, along with a subjective assessment of satiety. Methods: Fifteen overweight/obese subjects aged 18 - 45 years were recruited. After overnight fasting, blood samples were drawn at 5 mins before food consumption (-5), 0, 15, 30, 45, 60, 90 and 120 minutes. Participants underwent 3 days of reference food testing and 1 day of test food in random order with 2 days of wash-out period. The GI was assessed using a validated protocol recognized by $\mathrm{FAO} / \mathrm{WHO}$, as well as the guidelines by the International Dietary Carbohydrate Task Force for GI Methodology. The satiety index was measured using the Visual Analog Scale (VAS). The dietary intake of the subjects was measured by 24-hour dietary recall. The Incremental Area Under the Curve (IAUC) was calculated using the trapezoid rule. Results: Both the flavours of the supplement had low GI \& GR. The GI and GR of Flavour 1 were $27.3 \pm 4.8 \& 16.4 \pm 2.6$ (Mean \pm SEM) respectively. For Flavour 2 the GI and GR were $36.7 \pm 4.4 \& 25.7 \pm 2.3$ (Mean \pm SEM). For Flavour 1, 60\% and for flavour $266.7 \%$ of subjects reported feeling hungry only after 3 hours, showing good satiety. Conclusion: The plant-based high fibre high protein supplement in both flavours showed a low glycemic index and hence may be useful to include in the diets to reduce the postprandial glycemic response and could improve satiety.
\end{abstract}




\section{Keywords}

Glycemic Index, High Protein, High Fiber, Satiety, Visual Analog Scale

\section{Introduction}

The prevalence and incidence of diabetes is increasing across the world. Diabetes has an economic impact along with its impact on individual health. Obesity is a well-known major independent risk factor for developing type 2 diabetes mellitus (T2DM) [1] and is strongly correlated with a reduction in insulin sensitivity, especially in people with excess abdominal fat distribution and physical inactivity [2]. The prevalence of diabetes in India is also on the rise. The prevalence of diabetes reported in 15 states of India was 7.3\% (95\% CI 7.0 - 7.5). The overall prevalence of pre-diabetes was $10.3 \%$ [3].

Diet plays a vital role in managing diabetes. The amount of carbohydrates, fiber, protein in the diet and available insulin may be the most important factor influencing the glycemic response after eating and should be considered when developing a diet plan. Monitoring carbohydrate intake, whether by carbohydrate counting or experience-based estimation, remains a key strategy in achieving glycemic control. Substituting low-glycemic load foods for higher-glycemic load foods may modestly improve glycemic control [4]. The concept of Glycemic Index (GI) has clinically important benefits for preventing, managing, and treating a number of chronic diseases such as diabetes, cardiovascular disease (CVD), and obesity [5]. The GI is defined as the incremental area under the blood glucose response elicited by 25 or $50 \mathrm{~g}$ of available carbohydrate containing test food portion expressed as a percentage of the response to 25 or 50 $\mathrm{g}$ of glucose taken by the same participant [6]. It compares mass to mass of carbohydrates in single foods, while "Glycemic Response" (GR) is the term to be used while describing foods containing more than one source of carbohydrates (example: Mixed meals, breakfast cereals with milk, beverage mix with milk). Foods that are classified as low GI elicit a lower increment in blood glucose levels, with only slight increases in circulating levels of insulin and gastrointestinal hormones. Therefore, satiety is increased and voluntary food intake is reduced [7].

Foods with high satiety value would be helpful in reducing subsequent meal intake and thereby reduce energy intake, compared to foods with low satiety value. Foods that induce greater satiety could also aid in weight management, which is of paramount importance in individuals with T2DM. While studies have shown an inverse association between satiety and GI values, satiety is also dependent on the amount of macronutrients and dietary fibre content in addition to the sensory perceptions of the consumer. Hence, satiety is dependent on more than one component of the food and an integrated approach is needed in developing foods with higher satiety [8]. Keeping this background in mind, the 
objective of the study was to evaluate the glycemic Index, glycemic Response and satiety value of a high protein high fibre nutritional supplement.

\section{Methodology}

\subsection{Subjects}

Fifteen overweight/obese subjects aged 18 - 45 years were recruited to participate in the study. Subjects on special diets, with self-reported diabetes, suffering from any chronic illness or allergy, or on regular medications which could influence blood glucose levels, digestion and absorption were excluded from the study.

\subsection{Test and Reference Food}

Test food was a high protein high fibre (HPHF) nutritional supplement supplied by Dr. Reddy's Laboratories. As per the CODEX guidelines [9], if the product has $12 \%$ of protein, we can claim it as a high protein product. The amounts of test food for dietary GI study (each containing $25 \mathrm{~g}$ available carbohydrates) were as follows:

1) $134 \mathrm{~g}$ of nutritional supplement (flavor 1) mixed in $670 \mathrm{~mL}$ of water

2) $153 \mathrm{~g}$ of nutritional supplement (flavor 2) mixed in $765 \mathrm{~mL}$ of water

The test foods for glycemic response (GR) study (each containing $25 \mathrm{~g}$ available carbohydrates) were as follows:

1) $46 \mathrm{~g}$ of nutritional supplement (flavor 1) mixed in $300 \mathrm{~mL}$ of milk

2) $38 \mathrm{~g}$ of nutritional supplement (flavor 2) mixed in $345 \mathrm{~mL}$ of milk

Reference food was $27.5 \mathrm{~g}$ of monohydrate glucose dissolved in $100 \mathrm{~mL}$ of water for both GI and GR.

\subsection{The Procedure of Determining GI}

All subjects underwent 3 days of testing with the reference food and 1 day with the test food in random order with at least 2 days washout period between measurements to minimize carry-over effects.

Subjects visited the center each test day in the morning after a $10-12 \mathrm{hr}$ overnight fast. 24-hour dietary recall and details on physical activity, smoking, alcohol and caffeine-containing drinks were obtained to ensure that the subjects maintained the same diet and physical activity on pre-test dates and refrained from smoking and alcohol during the study period. Female subjects were not tested during menstrual period dates; testing was rescheduled in such cases. Fasting blood samples were taken at 5 mins before and immediately preceding consumption of the food for assessment of blood glucose levels by finger-prick using an automatic lancet device [Onetouch ${ }^{\circledR}$ UltraSoft Lancing Device (One-Touch ${ }^{\circledR}$ Ultra $^{\circledR} 2$, LifeScan, Livingstone, UK)], and the baseline value was taken as the mean of these two values. Further blood samples were taken at 15, $30,45,60,90$, and $120 \mathrm{~min}$ after starting to eat. The third finger on the left hand was used for all finger-prick blood samples. Before the finger prick, the subjects were encouraged to warm their hands to increase blood flow. Care was taken not 
to extract blood from the fingertip by squeezing, so as to avoid diluting the blood with plasma. A $0.6 \mu \mathrm{L}$ blood sample was used to measure the blood glucose using the Hemocue Glucose analyser 201+. The blood glucose meters were calibrated daily using control solutions from the manufacturer.

The study was conducted as per the GI testing protocol recognized by FAO/WHO, 1998 [10] as well as the guidelines by the International Dietary Carbohydrate Task Force for GI Methodology [11] and ISO [12] which have been validated and published elsewhere.

\subsection{Satiety}

The test foods were provided to participants between $4.00 \mathrm{pm}-5.00 \mathrm{pm}$ in random order with at least 2 days gap as a washout period between each test food. Satiety index rating using the visual analogue scale (VAS) was performed soon after the complete ingestion of the test food and also when a participant reported 'feeling hungry' after the consumption of each test food. The attributes on the VAS included with the score indicated-extremely hungry (-3), hungry $(-2)$, semi hungry (-1), no particular feeling (0), semi satisfied (1), satisfied (2), extremely satisfied (3). The dietary intake on the day prior to the test and on the day of the test was assessed using 24-hour dietary recall. During the entire study period, all the participants were advised not to alter any other aspects of their lifestyle.

\subsection{Statistical Analysis}

Fifteen overweight/obese individuals participated in the study. Out of the 3 reference values, one outlying value was removed with $\mathrm{CV}>30 \%$. Individuals with mean GI or GR $> \pm 2 \mathrm{SD}$ (unrepresentative response) were considered as outliers. Hence, for both GI and GR, the average of 2 reference food values was considered for 4 subjects whose CV were $>30 \%$; For GR, one subject from nutritional supplement flavour-1 with milk and 2 volunteers from nutritional supplement flavour-2 with milk were removed as outliers and therefore not included in the analysis. The Incremental Area under the Curve (IAUC) of blood glucose for the reference and test food were calculated geometrically using the trapezoid rule, ignoring the area below the fasting baseline. The mean and standard errors (SEM) of the IAUC for the reference and test food were calculated. GI and GR values were calculated by expressing each subject's IAUC after the test food as a percentage of the same subject's mean reference IAUC. The mean of the resulting values was taken as the GI and GR of the respective test food. The GI and GR values were further tested to see the influence (interaction) by age (yrs), sex, diet [energy (kcal), protein $(\mathrm{g})$, fat $(\mathrm{g})$, carbohydrates $(\mathrm{g})$, and dietary fibre $(\mathrm{g})$ ] and physical activity level (PAL) using General Linear Model (GLM).

$\mathrm{GI}^{1,2} / \mathrm{GR}$ value of test food(\%)

$$
=\frac{\text { Blood glucose IAUC value for the test food }}{\text { IAUC value of the reference food }} \times 100
$$




\subsection{Ethical Considerations}

Subjects were given full details of the study protocol and the opportunity to ask questions. The procedure used in this study was in accordance with international standards for conducting ethical research with humans and was approved by the Institutional Ethics Review Committee and all volunteers gave written informed consent to participate in the study. The study was registered in the clinical trial registry of India, CTRI/2018/04/012979.

\section{Results and Discussion}

The baseline characteristics of the study participants are given in Table 1. The individual GI and GR values and the standard error of mean (SEM) for the test foods were as follows: GI of the supplement with flavour-1 with water was $27.3 \pm$ 4.8 (Mean \pm SEM), flavour-2 with water $36.7 \pm 4.4$ (Mean \pm SEM). GR of supplement (flavour 1) with milk was $16.4 \pm 2.6$ (Mean \pm SEM), while that of supplement (flavour 2) with milk was $25.7 \pm 2.3$ (Mean \pm SEM) [Figure 1(a) \& Figure 1(b)]. The GI for both the flavours was not significantly different $(\mathrm{P}>$ 0.05). The GI and GR values were not influenced by age (yrs), sex, diet [energy (kcal), protein (g), fat (g), carbohydrates (g), and dietary fibre (g)] and physical activity level.

The GI of foods is influenced by many factors like the content of fat, protein and dietary fibre, cooking method, chewing time and chemical structure of the main carbohydrate. Further, the GI of a food differs when eaten alone and when consumed along with other foods. Fat, protein and dietary fibre tend to lower the GI of a food.

One of the systematic review and meta-analysis have shown that low-GI diets are more effective in controlling $\mathrm{HbAlc}$ and fasting blood glucose compared with higher-GI diets or control diets in patients with type 2 diabetes [13]. There has been an increase in the consumption of refined foods and simple sugars in

Table 1. Baseline characteristics of study participants.

\begin{tabular}{cc}
\hline Characteristics & Mean \pm SD \\
\hline Age (years) & $31.7 \pm 7.7$ \\
Female $(\mathrm{n} \%)$ & $8(53.3 \%)$ \\
Male $(\mathrm{n} \%)$ & $7(46.7 \%)$ \\
Height $(\mathrm{cm})$ & $162 \pm 12$ \\
Weight $(\mathrm{kg})$ & $69.3 \pm 6.8$ \\
Body mass index $\left(\mathrm{kg} / \mathrm{m}^{2}\right)$ & $26.5 \pm 3.2$ \\
Waist circumference $(\mathrm{cm})$ & $87.1 \pm 5.5$ \\
Systolic blood pressure $(\mathrm{mmHg})$ & $115 \pm 12.0$ \\
Diastolic blood pressure $(\mathrm{mmHg})$ & $75 \pm 4$ \\
Fasting blood glucose $\mathrm{mg} / \mathrm{dl}$ & $86 \pm 9$ \\
\hline
\end{tabular}




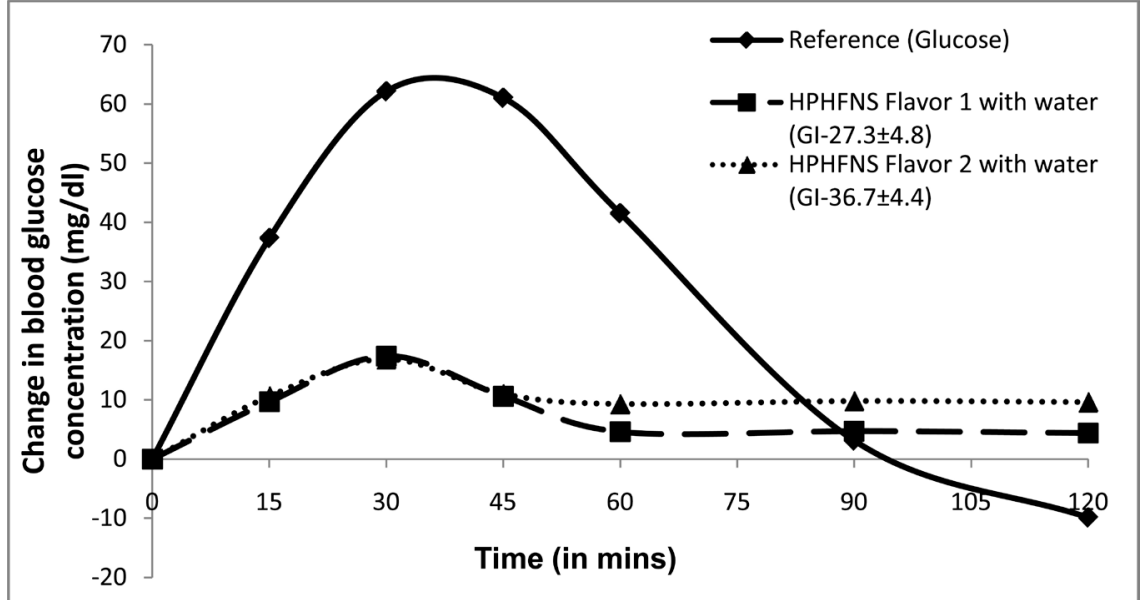

(a)

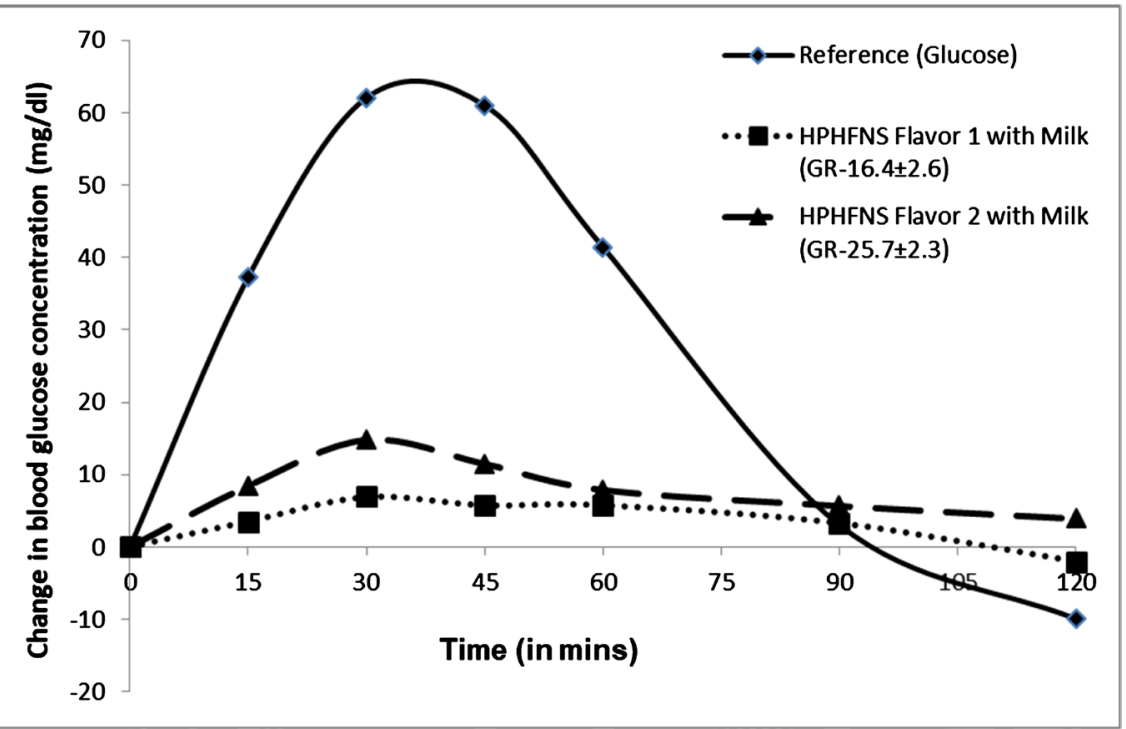

(b)

Figure 1. (a) Glycemic Index (GI) of both the nutritional supplement compared to reference food; (b) Glycemic Response (GR) of both the nutritional supplement compared to reference food.

place of complex carbohydrates. Therefore, to contain the pandemic rise of this metabolic disease, the consumption of complex carbohydrates with a low glycemic index (GI) is very important. In India, where the fiber and the protein intake is low, high fibre and protein nutritional supplements have to become part of the routine diet to control the overall impact on blood sugar levels [10].

Satiety ratings of the products tested are given in Table 2 . Overall, all the 4 test foods showed similar satiety related ratings (response). After consumption of flavour 2 of drink with either water or milk, $60 \%$ of subjects felt "Semi hungry". For flavour 1 with water, $47 \%$ of the subjects felt "semi hungry" and with milk, 53\% felt "semi hungry". This "semi hungry" response was reported 3.1 hours post-ingestion of nutritional supplement flavour-1 with water before the next meal/drink, whereas for nutritional supplement flavour-1 with milk, the 
Table 2. Satiety rating for the test products.

\begin{tabular}{cccccc}
\hline Test Foods & $\begin{array}{c}\text { Overall Hungry } \mathbf{n}(\%) \\
\text { (semi hungry and hungry) }\end{array}$ & $\begin{array}{c}\text { Hungry } \\
\mathbf{n}(\%)\end{array}$ & $\begin{array}{c}\text { Semi Hungry } \\
\mathbf{n}(\%)\end{array}$ & $\begin{array}{c}\text { No Particular Feeling } \\
\mathbf{n}(\%)\end{array}$ & $\begin{array}{c}\text { Satisfied } \\
\mathbf{n}(\%)\end{array}$ \\
\hline HPHF Flavour 1 with water & $9(60.0 \%)$ & $2(13.3 \%)$ & $7(46.7 \%)$ & $5(33.3 \%)$ & $1(6.7 \%)$ \\
HPHF Flavour 1 with Milk & $10(66.7 \%)$ & $2(13.3 \%)$ & $8(53.3 \%)$ & $4(26.7 \%)$ & $1(6.7 \%)$ \\
HPHF Flavour 2 with water & $11(73.3 \%)$ & $2(13.3 \%)$ & $9(60.0 \%)$ & $3(20.0 \%)$ & $1(6.7 \%)$ \\
HPHF Flavour 2 with Milk & $11(73.3 \%)$ & $2(13.3 \%)$ & $9(60.0 \%)$ & $3(20.0 \%)$ & $1(6.7 \%)$ \\
\hline
\end{tabular}

duration was reduced to 2 hours. However, flavour 2 with either milk or water provided satiation for just 1.6 to 1.9 hours among those who reported "semi hungry". The "overall hungry" satiety rating was derived from the average of "hungry" and "semi hungry" responses reported in the VAS. Almost two-thirds of the study subjects (60\%) reported an "overall hungry" rating after 3 hours of ingestion of flavour 1 with milk, while $66.7 \%$ reported this rating after 2.3 hours. Although almost three-fourths of the study subjects (73\%) reported feeling "overall hungry" for flavour 2 with water and milk, the average duration being 1.7 hours for flavour 2 with milk and 2.3 hours for flavour 2 with water.

There was only 1 subject who reported "satisfied" (feeling full) for almost 5 hours for flavour 2 with milk whereas another subject reported feeling "satisfied" [but only for 1.3 hours], as the subjects used to take evening beverage of soup at this hour. Soon after the ingestion of test foods, $75 \%$ of the subjects reported feeling "semi satisfied".

Satiety is influenced by many factors. It involves brain, body, social and physical environment. Subjects would be benefited from controlling their appetite, eating healthy and maintaining or reducing body weight by consumption of foods that provide satiety [14]. In between snacking during the day is one of the major factors for weight management. If the snack does not provide satiety, it just adds to the calories which a person consumes and also impacts the intake of the next meal. Therefore, it is important to identify healthy snacks which provide satiety and support appropriate calorie dependent adjustments of subsequent intake, so that snacking is less likely to result in a net increase in energy consumed [15]. Evening snack intake of the study participants showed significant changes in energy (kcal), carbohydrates (g) and protein (g) content following intake of flavour 1 with water whereas only carbohydrate (g) and protein (g) content changed following intake of flavour 1 with milk. Following intake of flavour 2 with water, there was a significant difference in the carbohydrates (g) content of the evening snack while there was no significant difference in diet data following the intake of flavour 2 with milk.

Reducing energy intake while maintaining a healthy eating pattern with high protein and fibre is recommended to promote weight loss. Even modest weight loss may provide clinical benefits (improved glycemia, blood pressure and or 
lipids.) In some individuals with diabetes, especially those in early stages, substituting low glycemic load foods for higher glycemic load foods may modestly improve glycemic control [16].

According to Bronus et al., the subject characteristics do not appear to have a significant effect on mean GI values but the variation of the values may differ in various groups, being highest in individuals suffering from type 1 diabetes. Therefore, in routine testing, healthy human subjects are recommended. Further studies in diabetic subjects would further support these findings [17]. Weight loss is an additional potential mechanism by which low-GI diets may contribute to a reduced risk of metabolic syndrome. Induction of a rapid initial weight loss with low-carbohydrate diets may be partly explained by a reduction in overall caloric intake, which may be the result of a great limitation of food choices by the requirements of minimizing carbohydrates intake [18] [19], to the initial increase in circulating $\beta$ hydroxybutyrate, which may suppress appetite [20] and to the satiating effect of low carbohydrates diets containing relatively high amounts of protein [21] [22].

\section{Conclusions}

The Glycemic Index for both the flavours is below 55 and therefore has a low GI. The product is scientifically designed to have high fibre and high protein. The protein and fibre content of the product contributes to the low GI. The glycemic response of the product is also low when consumed with milk, as is its glycemic load.

Use of this product along with regular meal is likely to be of help in individuals with diabetes as it decreases the flux of glucose released in the bloodstream after digestion. This will not only help in reducing the postprandial glucose spikes in these individuals, but will also enable them to control their calorie intake through induction of satiety. Still, larger studies involving subjects with diabetes, looking at the endpoints like the impact on glucose excretion, HbA1C, satiety and its impact on weight management are needed to delineate the place of such nutritional supplements in the diabetologist's armamentarium.

\section{Conflicts of Interest}

The authors declare no conflicts of interest regarding the publication of this paper.

\section{References}

[1] Steinberger, J. and Daniels, S.R. (2003) Obesity, Insulin Resistance, Diabetes, and Cardiovascular Risk in Children: An American Heart Association Scientific Statement from the Atherosclerosis, Hypertension, and Obesity in the Young Committee (Council on Cardiovascular Disease in the Young) and the Diabetes Committee (Council on Nutrition, Physical Activity, and Metabolism). Circulation, 107, 1448-1453. https://doi.org/10.1161/01.CIR.0000060923.07573.F2

[2] Hensrud, D. (2001) Dietary Treatment and Long-Term Weight Loss and Mainte- 
nance in Type 2 Diabetes. Obesity Research, 9, 348S-353S.

https://doi.org/10.1038/oby.2001.141

[3] Anjana, R.M., Deepa, M., Pradeepa, R., Mahanta, J., Narain, K., Das, H.K., et al. (2017) Prevalence of Diabetes and Prediabetes in 15 States of INDIA: Results from the ICMR-INDIAB Population-Based Cross-Sectional Study. The Lancet Diabetes \& Endocrinology, 5, 585-596.

[4] Evert, A.B., Boucher, J.L. Cypress, M., Dunbar, S.A., Franz, M.J., Mayer-Davis, E.J., et al. (2013) Nutrition Therapy Recommendations for the Management of Adults with Diabetes. Diabetes Care, 36, 3821-3842.

[5] Martin, C.L., Murphy, S.P. and Au, D.L.M. (2008) Compiling Glycemic Index and Glycemic Load Values for Addition to a Food Composition Database. Journal of Food Composition and Analysis, 21, 469-473.

https://doi.org/10.1016/j.jfca.2008.03.007

[6] Wolever, T.M., Vorster, H.H., Björck, I., Brand-Miller, J., Brighenti, F., Mann, J.I., Ramdath, D.D., Granfeldt, Y., Holt, S., Perry, T.L., Venter, C. and Wu, X.-M. (2003) Determination of the Glycaemic Index of Foods: Interlaboratory Study. European Journal of Clinical Nutrition, 57, 475-482. https://doi.org/10.1038/sj.ejcn.1601551

[7] Aller, E.E.J.G., Abete. I., Astrup, A., Martinez, J.A. and van Baak, M.A. (2011) Starches, Sugars and Obesity. Nutrients, 3, 341-369.

https://doi.org/10.3390/nu3030341

[8] Chambers, L., McCrickerd, K. and Yeomans, M.R. (2015) Optimising Foods for Safety. Trends in Food Science \& Technology, 41, 149-160. https://doi.org/10.1016/j.tifs.2014.10.007

[9] Guidelines for Use of Nutrition and Health Claims (CAC/Gl 23-1997).

[10] FAO (1998) Carbohydrates in Human Nutrition. Report of a Joint FAO/WHO Expert Consultation. FAO Food and Nutrition Paper, 66, 1-140.

[11] International Standards Organisation (2010) ISO 26642-2010: Food Products: Determination of the Glycaemic Index (GI) and Recommendation for Food Classification.

[12] Buyken, A.E., Mela, D.J., Dussort, P., Johnson, I.T., MacDonald, I.A., Stowell, J.D. and Brouns, F.J.P.H. (2018) Dietary Carbohydrates: A Review of International Recommendations and the Methods Used to Derive Them. European journal of clinical nutrition, 72, 1625-1643. https://doi.org/10.1038/s41430-017-0035-4

[13] Ojo, O., Ojo, O.O., Adebowale, F. and Wang, X.-H. (2018) The Effect of Dietary Glycaemic Index on Glycaemia in Patients with Type 2 Diabetes: A Systematic Review and Meta-Analysis of Randomized Controlled Trials. Nutrients, 10, Article ID: 373. https://doi.org/10.3390/nu10030373

[14] Blundell, J.E. and Tremblay, A. (1995) Appetite Control and Energy (Fuel) Balance. Nutrition Research Reviews, 8, 225-242. https://doi.org/10.1079/NRR19950014

[15] Halford, J.C. and Harrold, J.A. (2012) Satiety-Enhancing Products for Appetite Control: Science and Regulation of Functional Foods for Weight Management. Proceedings of the Nutrition Society, 71, 350-362. https://doi.org/10.1017/S0029665112000134

[16] Fabricatore, A.N., Wadden, T.A., Ebbeling, C.B., Thomas, J.G., Stallings, V.A., Schwartz, S. and Ludwig, D.S. (2011) Targeting Dietary Fat or Glycemic Load in the Treatment of Obesity and Type 2 Diabetes: A Randomized Controlled Trial. Diabetes Research and Clinical Practice, 92, 37-45. https://doi.org/10.1016/j.diabres.2010.12.016

[17] Brouns, F., Bjorck, I., Frayn, K.N., Gibbs, A.L., Lang, V., Slama, G. and Wolever, 
T.M.S. (2005) Glycaemic Index Methodology. Nutrition Research Reviews, 18, 145-171. https://doi.org/10.1079/NRR2005100

[18] Brehm, B.J., Seeley, R.J., Daniels, S.R. and D'alessio, D.A.A. (2003) Randomized Trial Comparing a Very Low Carbohydrate Diet and a Calorie-Restricted Low-Fat Diet on Body Weight and Cardiovascular Risk Factors in Healthy Women. The Journal of Clinical Endocrinology \& Metabolism, 88, 1617-1623. https://doi.org/10.1210/jc.2002-021480

[19] Sondike, S.B., Copperman, N. and Jacobson, M.S. (2003) Effects of a Low-Carbohydrate Diet on Weight Loss and Cardiovascular Risk Factor in Overweight Adolescents. The Journal of Pediatrics, 142, 253-258. https://doi.org/10.1067/mpd.2003.4

[20] Meckling, K.A., Gauthier, M., Grubb, R. and Sanford, J. (2002) Effects of a Hypocaloric, Low-Carbohydrate Diet on Weight Loss, Blood Lipids, Blood Pressure, Glucose Tolerance, and Body Composition in Free-Living Overweight Women. Canadian Journal of Physiology and Pharmacology, 80, 1095-1105.

https://doi.org/10.1139/y02-140

[21] Johnston, C.S., Tjonn, S.L. and Swan, P.D. (2004) High-Protein, Low-Fat Diets are Effective for Weight Loss and Favorably Alter Biomarkers in Healthy Adults. The Journal of Nutrition, 134, 586-591. https://doi.org/10.1093/jn/134.3.586

[22] Layman, D.K., Boileau, R.A., Erickson, D.J., Painter, J.E., Shiue, H., Sather, C. and Christou, D.D. (2003) A Reduced Ratio of Dietary Carbohydrate to Protein Improves Body Composition and Blood Lipid Profiles during Weight Loss in Adult Women. The Journal of Nutrition, 133, 411-417.

https://doi.org/10.1093/jn/133.2.411 